
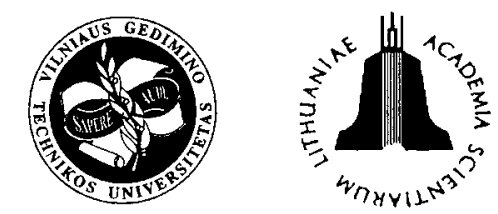

ISSN 1392-3730

JOURNAL OF CIVIL ENGINEERING AND MANAGEMENT

http:/www.vtu.lt/english/editions

2002, Vol VIII, No 4, 281-285

\title{
SELECTION OF THERMOPHYSICAL PROPERTIES OF HEAT STORAGE WALL
}

\author{
Halina Koczyk \\ Division of Heating, Air Conditioning and Air Protection, Poznan University of Technology, \\ ul. Piotrowo 3a, 60-965 Poznań, Poland. E-mail: Halina.Koczyk@put.poznan.pl
}

Received 20 Feb 2001; accepted 12 Sept 2002

\begin{abstract}
The paper discusses a method of the multilayered heat storage wall. Initial data: walls are multilayered, heat conductivity process is considered being one-dimensional, climatic factors and power supplied are periodic functions of time. In the paper mathematical and numerical models are presented. A mathematical model is constituted of a system of differential equations which describe heat conduction in material layers, equation of heat balance and boundary conditions system. Solving equations with regard to unknown property which has been recognized as a crucial parameter in the task one can obtain a real numeric value of the parameter.

The paper is illustrated by the nomograms of calculated accumulating layer thickness and the scheme of an algorithm designed to calculate the thickness of accumulating layer.
\end{abstract}

Keywords: thermal energy storage, heat storage wall, intermittent heating, heat transfer in a building, matrix method, numerical model, mathematical model.

\section{Introduction}

Thermal energy storage (TES) is very important in many engineering applications. For example, among the practical problems involved in solar energy systems is the need for an effective means by which the excess heat collected during periods of bright sunshine can be stored, preserved and later released for utilization during the night or other periods $[1,2]$.

The basic types of TES techniques are as follows:

- sensible heat storage,

- latent heat storage,

- thermochemical heat storage.

The selection of TES systems is mainly dependent on the storage period required, ie diurnal or seasonal (short and long-term storage), economical viability, operating conditions, etc. Heat storage can also be applied in most types of buildings where heating needs are significant and electricity rates allow heat storage to be competitive with other forms of heating.

Electrical consumption varies greatly during the day and the night. Such variation leads to differences in the electricity price during the off-peak period (usually from evening to morning), when it is reduced to approximately one-third or even one-fourth. Therefore, it is important to cut the electrical cost for space heating if the peak load could be shifted to the off-peak period. Commonly, a variation of TES is used to shift energy loads to offpeak periods in order to reduce utility charges and minimise or avoid high, on-peak demand charges [3, 4].
There are also some heating systems, which combine unconventional solar system and electrically heated TES systems [5-8]. The paper investigates the use of electrically heated heat storage wall (HSW) in order to reduce the heating cost, while retaining heat comfort in the room. It also aims at presenting a method of dimensioning the multilayered HSW. The method assumes a short-term storage in cycle of 24 hours.

To properly handle the problem, there is a need to solve the thermal model of the building incorporating a quasistationary, electrically heated HSW (as an element of the room enclosure). The HSW is loaded with energy cyclically (during off-peak periods).

The modelling of thermal behaviour of buildings has been the object of many studies [7-11]. A thorough inspection of widely used models, as well as of their corresponding algorithms is given in [12].

In this paper, both mathematical and numerical approximation models are presented for this purpose. The article was based on a developed computational model, using the matrix method for periodically varying heat extortions $[13,14]$. With reference to the room, the inverse internal problem of the first kind leading to the determination of thermophysical properties of the heat storage wall has been formulated.

\section{Problem formulation}

The question under consideration is heat transfer in a room with regular intermittent heating, from the source 


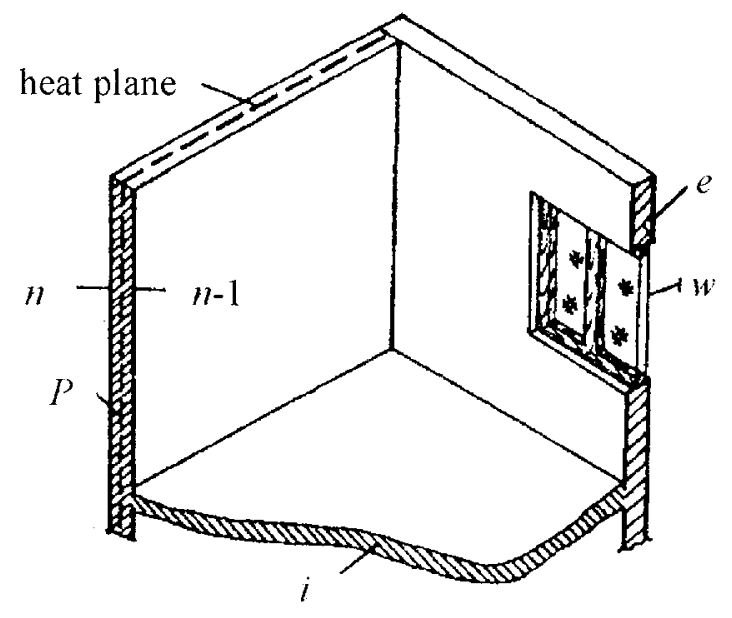

Fig 1. Fragment of the room enclosure with heat plane wall $\mathrm{HSW}$

of heat located in a HSW. A fragment of the room enclosure is shown in Fig 1.

The following assumptions have been made:

- walls forming the enclosure are assumed to be multilayered walls,

- one of the walls is supplied with electric energy in off-peak periods,

- heat conduction is considered as being one-dimensional disregarding corners, thermal bridges and window framing,

- physical properties of built-in materials of the walls are constants,

- air temperature is assumed to be the same in all room's volume,

- heat transfer on internal surfaces of the walls takes place with constant heat convection coefficients,

- time courses of internal air temperature in adjacent rooms are identical,

- climatic factors and thermal power are periodic functions of time.

By assuming periodicity it is possible to disengage the model from the influence of initial conditions. A mathematical model of a room [1-3] constitutes a system of parabolic Fourier-Kirchhoff partial differential equations which describe heat conduction in material layers (the number of equations corresponds with the total number of layers in walls forming the enclosure). It is completed by the equation of heat balance of the room air, as well as, the boundary conditions system. The problem of selecting heat storage wall can be formulated as the inverse internal problem of the first kind.

It is necessary to calculate thermophysical parameters of heat storage wall assuring, in given energy supply conditions, the required $24 \mathrm{~h}$ course of internal air temperature $f(\tau)$ for the defined surface elements forming the room enclosure and defined construction of all elements, apart from the heater.
Time function of internal air temperature is determined by introducing a pair of numbers: $t_{i m}$ - mean 24 $\mathrm{h}$ course temperature of internal air, $\delta t_{i m}$ - mean square deviation

$$
\begin{gathered}
t_{i m}=\frac{\int_{0}^{\tau_{0}} f(\tau) d \tau}{\tau_{0}} \\
\delta t_{i m}=\frac{\sqrt{\int_{0}^{\tau_{0}}\left[t_{i m}-f(\tau)\right]^{2} d \tau}}{\tau_{0}}
\end{gathered}
$$

$f(\tau)$ - function of internal air temperature variation obtained by solving the room model,

$\tau_{0}-$ period of function variation, $\tau_{0}=24 \mathrm{~h}$.

The object of selection can be any parameter of heat storage wall, eg thickness of the accumulating layer, heat conduction coefficient, mass density, etc. On the basis of engineering practice it has been assumed that thickness of the accumulating layer is a crucial parameter in this task.

\section{Numerical model of a room with the HSW}

In selecting heat storage wall a numerical model of a room based on the matrix method has been used. The functions of a numeric model are as follows:

- determination of input functions of the model, eg functions of external climate factors and productiveness of heat source, their harmonic analysis and placing them in a disk file, the so-called "process catalogue",

- calculating harmonic components of the walls catenary matrix and putting them in a disk file, the socalled "walls catalogue",

- calculating catenary matrix harmonic components of the accumulating heater,

- construction and solution of a numeric model of a room including calculation of harmonic components on internal walls surfaces as well as computation of harmonic components of the air temperature inside, harmonic synthesis of $24 \mathrm{~h}$ course of internal air temperature, determination and investigation of deviation,

- results output.

Basic equations for heat balance of the room thermal centre is derived from the relation

$$
\left[\begin{array}{l}
\overrightarrow{t_{e j, k}} \\
\overrightarrow{q_{e j, k}}
\end{array}\right]=\left[\begin{array}{ll}
A_{j, k} & B_{j, k} \\
C_{j, k} & D_{j, k}
\end{array}\right] \cdot\left[\begin{array}{l}
\overrightarrow{t_{i j, k}} \\
\overrightarrow{q_{i j, k}}
\end{array}\right]
$$

where

$A, B, C, D$ - designate elements of the catenary matrix of the $j$-th wall for the $k$-th harmonic,

$\overrightarrow{t_{i j, k}}$ - the $k$-th harmonic component of temperature inside the $j$-th wall, 
$\overrightarrow{t_{e j . k}}$ - the $k$-th temperature component of temperature outside the $j$-th wall,

$\overrightarrow{q_{i j, k}}$ - the $k$-th harmonic component of heat flux density inside the $j$-th wall,

$q_{e j, k}$ - the $k$-th harmonic component of heat flux density outside the $j$-th wall.

Elements of the wall catenary matrix are obtained by postmultiplication of the catenary matrix of particular material layers and matrices including surface film conductances. The catenary matrix of the material layer with $d$ - thickness and $s$ - heat assimilation coefficient has the following elements:

$$
\begin{aligned}
& A=D=\cosh \frac{d s \cdot \sqrt{i}}{\lambda}, \\
& B=\frac{1}{s \cdot \sqrt{i}} \sinh \frac{d s \cdot \sqrt{i}}{\lambda}, \\
& C=s \cdot \sqrt{i} \cdot \sinh \frac{d s \cdot \sqrt{i}}{\lambda} .
\end{aligned}
$$

The heat assimilation coefficient of material layer depends on material thermophysical parameters $c, \rho, \lambda$ and $\omega$ angular velocity.

$$
s=\sqrt{c \cdot \omega \cdot \rho \cdot \lambda}
$$

where

$c$ - specific heat,

$\rho$ - material density,

$\lambda$ - thermal conduction,

$\omega$ - angular velocity, $\omega=2 \pi / \tau_{0}$.

The catenary matrix of the element with thermal resistance $R$ is expressed by:

$$
A=D=1 ; \quad B=R ; \quad C=0 .
$$

The catenary matrix of the element with thermal capacity $C$ is expressed by:

$$
A=D=1 ; \quad B=0 ; \quad C=\mathrm{C} \omega i .
$$

The elements with thermal resistance may be, eg air-gaps, planes of surface film conductance and planes of overall heat transfer. The elements with thermal capacity may be eg water volume of heating system, energy accumulator.

Owing to the catenary matrix, it is possible to determine temperature harmonic components and heat flux densities on the one wall side on the basis of their values on the other side.

The room shown in Fig 1 is considered as a set of walls and each of them is in contact with the air by an unknown course $t_{i}(\tau)$.

With regard to boundary conditions outside the model there can be distinguished:

- external walls " $e$ " for which the course of external air temperature $t_{e}(\tau)$ is given,

- external transparent walls (eg) windows " $w$ " for which the course of solar gain $Q_{S}(\tau)$ is given,
- internal walls " $i$ " located between rooms characterised by identical air temperature courses $t_{e}(\tau)=t_{i}(\tau)$

- internal symmetric filling " $s$ " located inside the zone for which the symmetry of construction and temperature distribution will be assumed,

- plane heater " $p$ " for which the function of productiveness of heat source defined in terms of time function $q_{p}(\tau)$ is given. Storage wall has been divided into two parts: " $n-1$ " and " $n$ " connected by a common heating plane.

The section of plane heater " $p "$ is shown in Fig 2.

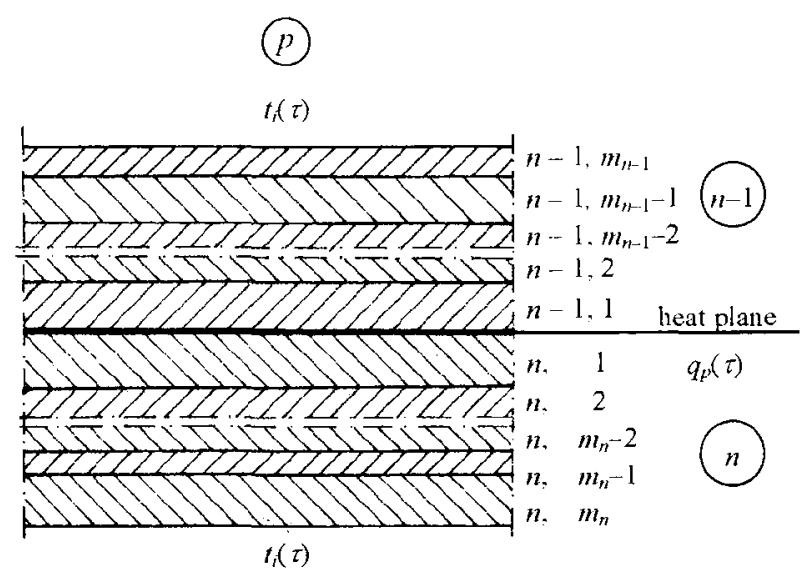

Fig 2. The section of plane heater

In relation to each harmonic component the heat balance equation of the air in the room should be solved.

$$
A_{i j} \cdot \overrightarrow{q_{i j, k}}+\overrightarrow{Q_{s k}}=0
$$

where

$$
\begin{aligned}
& A_{i j} \text { - internal surface of the } j \text {-th wall in the room, } \\
& \overrightarrow{q_{i j, k}}-\text { heat flux density on internal surface of the } \\
& \overrightarrow{Q_{s k}} \quad \text { - solar wall, }
\end{aligned}
$$

In the model ventilation is considered by introducing an additional external wall with a suitable catenary matrix. Heat flux densities on external wall surfaces $\overrightarrow{q_{i j, k}}$ can be determined from (3) accordingly taking into account boundary conditions on external wall surfaces with reference to each harmonic component separately, in succession:

- for external walls " $e "$

$$
\overrightarrow{q_{i j, k}}=\overrightarrow{-t_{i k}} A_{B_{j, k}}^{A_{j, k}}+\overrightarrow{t_{e k}} \begin{gathered}
1 \\
B_{j, k}
\end{gathered},
$$

- for internal walls " $i$ "

$$
\overrightarrow{q_{i j, k}}=\overrightarrow{-t_{i k}} \frac{A_{j, k}-1}{B_{j, k}},
$$


- for internal symmetric filling " $s "$

$$
\overrightarrow{q_{i j, k}}=\overrightarrow{-t_{i k}} \frac{C_{j, k}}{D_{j, k}},
$$

- for the heat storing walls " $p "$ - equations (12) and (13).

$$
\begin{aligned}
& \overrightarrow{q_{i j, k}}=A_{i p} \cdot \overrightarrow{t_{i k}} \cdot\left(\begin{array}{c}
2-\left(A_{n, k} \cdot D_{n-1, k}+A_{n-1 . k} \cdot D_{n, k}\right) \\
B_{n-1, k} \cdot D_{n, k}+B_{n, k} \cdot D_{n-1, k}
\end{array}+\right. \\
& \left.+\begin{array}{c}
-\left(B_{n, k} \cdot C_{n-1 . k}+B_{n-1, k} \cdot C_{n, k}\right) \\
B_{n-1, k} \cdot D_{n, k}+B_{n, k} \cdot D_{n-1, k}
\end{array}\right) .
\end{aligned}
$$

The component of solar heat gains is not taken into account in equation 8 .

From the equation 8 the harmonic component of internal air can be assigned.

Designations to be used are:

$e$ - external walls summation,

$i$ - internal walls summation,

$s$ - internal symmetric filling summation.

Determination of time function $t_{i}(\tau)$ is necessary for the harmonic synthesis.

\section{Method of dimensioning the HSW}

The equation (13) gives a single harmonic component of internal air temperature. If we take no account of the stationary harmonic component in the solution and make the harmonic synthesis of the function $t_{i}$, we will obtain $24 \mathrm{~h}$ course of internal air temperature deviations from the mean. From this course, following elementary operations, mean deviation of the internal air temperature $\delta t_{i m}$ can be determined. Thus, the deviation $\delta t_{i m}$ is given by a transcendential equation resulting from a complex consideration of (13) and the harmonic synthesis equations.

An unknown in the transcendential equation can be any chosen parameter of heat storing wall construction. Solving the equation with regard to unknown thickness of the accumulating layer, which has been recognised as a crucial parameter in the task, we obtain a real numeric value of the layer thickness above which no exceeding of the defined mean square deviation of $24 \mathrm{~h}$ course of internal temperature will occur.

In Fig 3 there is a scheme of an algorithm designed to calculate the thickness of the accumulating layer in a HSW. The mean square deviation of the course of air temperature inside the room was marked as $\delta$, and the accuracy of a solution as $\varepsilon$. An analogous algorithm may be used for determining selected thermophysical proper-

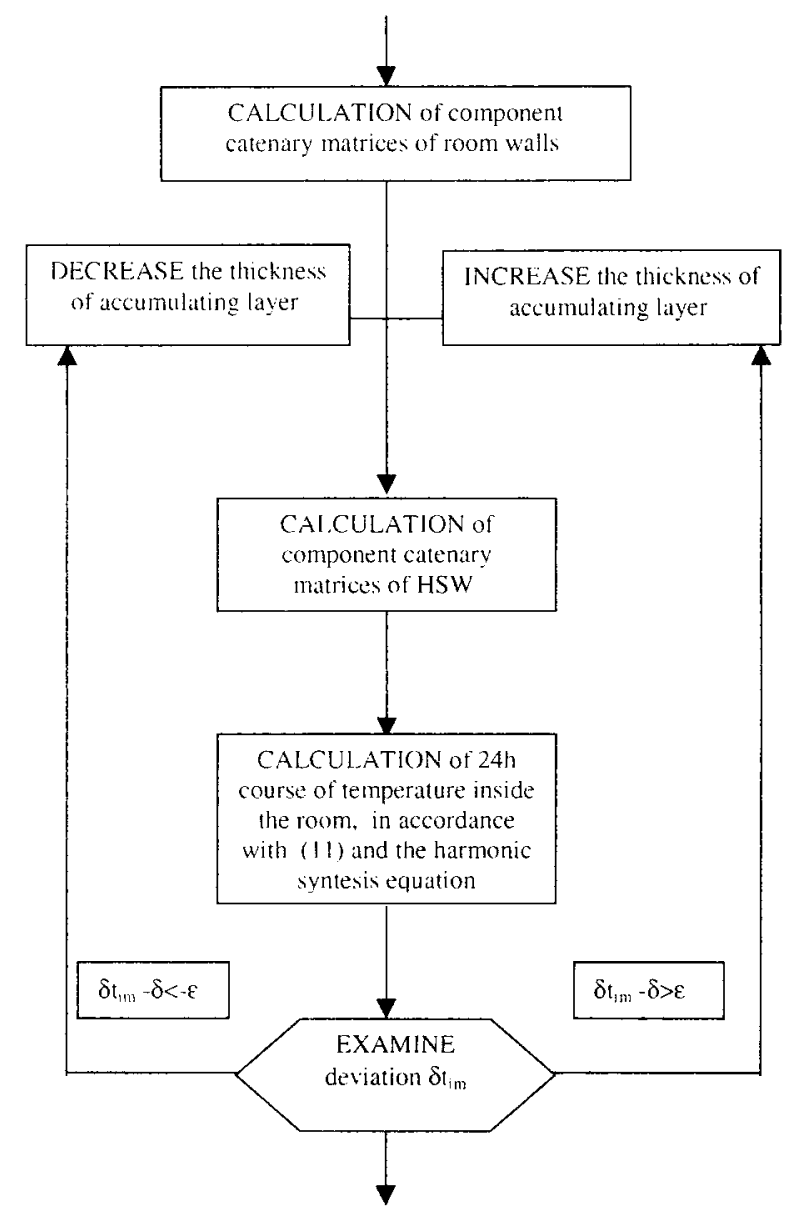

Fig 3. Scheme of an algorithm designed to calculate the thickness of the accumulating layer in a HSW

ties of a HSW, eg: density $\rho$, or heat capacity $c$ of the accumulating material.

Exemplary results of dimensioning according to the proposed method are presented in Fig 4. In the selection diagram the following have been used as primary coordinate axes: thickness of the accumulating layer $d_{\alpha c}[\mathrm{~cm}]$ and temperature $\mathrm{t}\left[{ }^{\circ} \mathrm{C}\right]$. Also three groups of curves characterising $24 \mathrm{~h}$ courses temperatures, namely maximal $24 \mathrm{~h}$ temperatures of the heater surface $t_{s_{1 \max }}$ and $t_{s_{2 \max }}$ and maximal temperature in heating plane $t_{p_{\max }}$ for different insulation thickness $d_{i n}$. It is necessary to know both maximal values of surfaces temperature $t_{s}$ in order to check requirements of heating comfort every time and the maximal heating plane temperature $t_{p}-$ in order to check construction requirements. Additionally, the diagrams have been supplied with isolines of mean deviations dtim obtained according to the presented method of selecting thickness of the accumulating layer.

$$
\overrightarrow{t_{i j, k}}=\frac{\overrightarrow{q_{p k}} \cdot A_{i p} \frac{B_{n-1, k}+B_{n, k}}{B_{n-1, k} \cdot D_{n, k}+B_{n, k} \cdot D_{n-1, k}}+\sum_{e} \overrightarrow{t_{e, k}} \cdot A_{i e} \cdot \frac{1}{B_{j, k}}}{A_{i j} \cdot \frac{\left(A_{n, k} \cdot D_{n-1, k}+A_{n-1, k} \cdot D_{n, k}+B_{n, k} \cdot C_{n-1, k}+B_{n-1, k} \cdot C_{n, k}-2\right.}{B_{n-1, k} \cdot D_{n, k}+B_{n, k} \cdot D_{n-1, k}}+\sum_{e} A_{i e} \cdot \frac{A_{j k}}{B_{j k}}+\sum_{i} A_{i i} \cdot \frac{A_{j, k}-1}{B_{j, k}}+\sum_{s} A_{i s} \cdot \frac{C_{j, k}}{D_{j, k}}}
$$




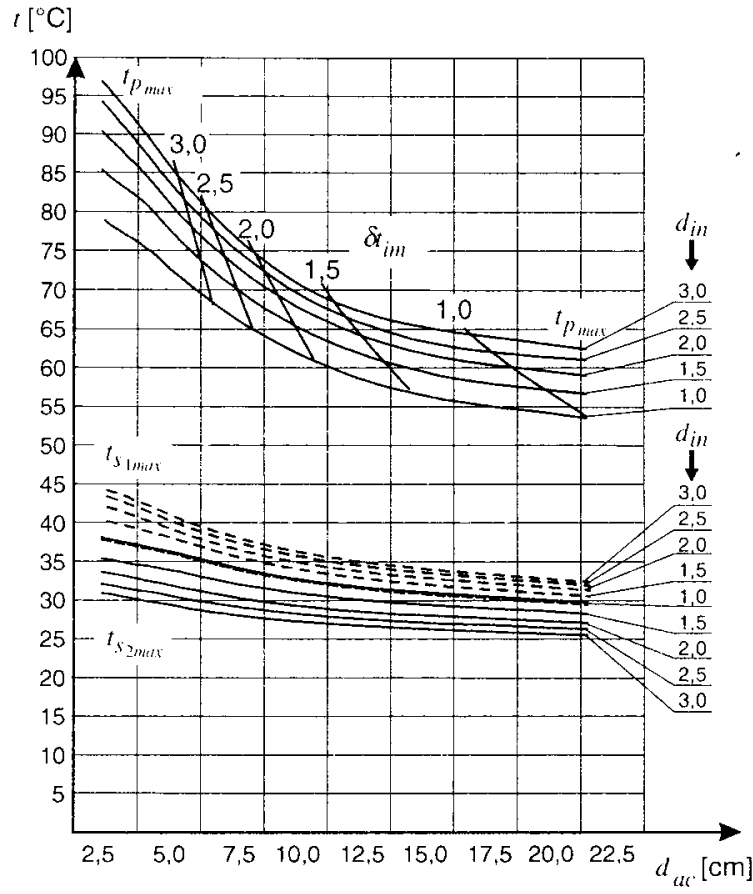

Fig 4a. Nomogram of thickness accumulating layer selection. Heating plane in the half-thickness

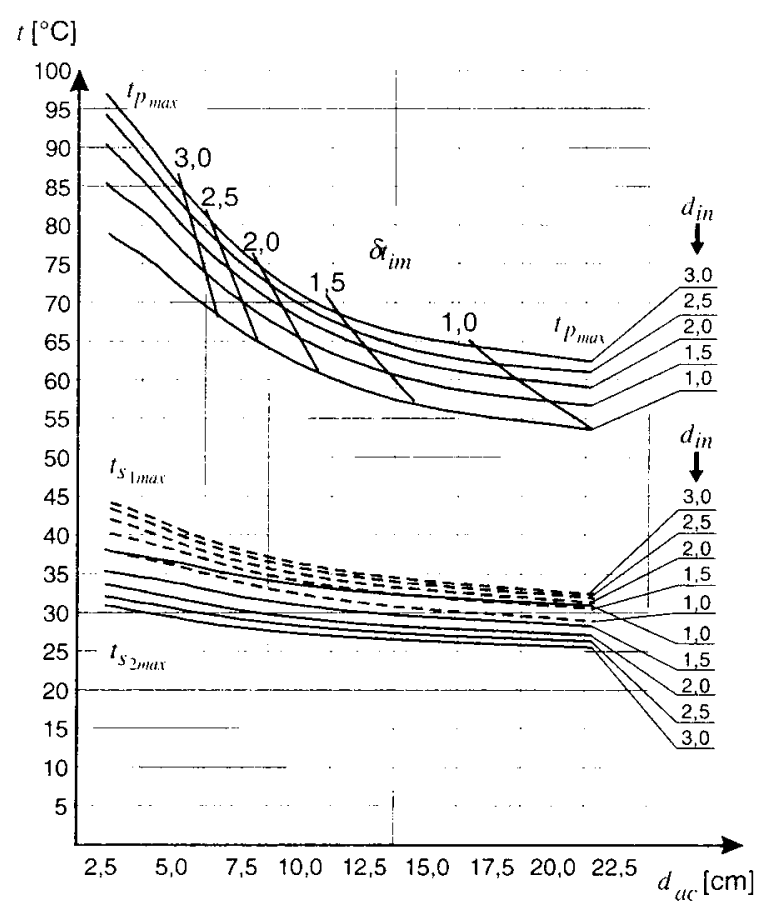

Fig 4b. Nomogram of thickness accumulating layer selection heating plane in the two-thirds thickness

There has also been considered the range of changes of the accumulating layer thickness of $1 \div 20 \mathrm{~cm}$ concrete and thickness changes of material insulation of $1 \div 3$ $\mathrm{cm}$ fibreboard. The position of heating plane has been assumed in the half-thickness of the accumulating layer (Fig 4a) or in the two-thirds thickness (Fig 4b).

\section{Conclusions}

As far as practical applications are concerned, the proposed nomograms constitute the full specification of data - for selecting electrically heated heat storage wall in a given group of design systems. They allow for alternative designing of the heater. After modification of the characteristic temperature axes, the diagrams can be used for analysing arrangement of the room with heat storage wall at different external air temperatures. The presented method can also be applied to other groups of accumulating heaters, supplied with energy periodically.

\section{References}

1. Duffie, J. A.; Beckman, W. A. Solar engineering of thermal processes. Wiley, New York, 1991

2. Hasnain, S. M. Review of sustainable thermal energy storage technologies. Part I. Heat storage materials and techniques. Energy, Conversion and Management, Vol 39, No 11, 1998, p 1127-1138.

3. Xiaoshiu, L.; Viljanen, M. Controlling building indoor temperature and reducing heating cost through night heating electric stove. Energy and Buildings, 33 (2001), p 865 873.

4. Braun, J. E. Reducing energy costs and peak electrical demand through optimal control of building thermal storage. ASHRAE Transactions, 96 (1990), p 876-888.

5. Bakos, G. C. Improved energy management method for auxiliary electrical energy saving in a passive-solar-heated residence. Energy and Buildings, 34, 2002, p 699-703.

6. Bakos, G. C. Energy management method for auxiliary electrical energy saving in a passive-solar-heated residence using low-cost off-peak electricity. Energy and Buildings, 31, 2000, p 237-241.

7. Kossecka, E. The effect of structure on dynamic thermal characterisitics of multilayer walls. Archives of Civil Engineering, 3, 1996.

8. Mohl, U. Heat transfer in buildings. Design and simulation., $H L H$, No 2, 1987, p 83-86 (in German).

9. Muncey, R. W. R. Heat transfer calculations for buildings. Applied Science Publishers Ltd., London, 1979.

10. Sodha, M. S.; Kaur, J.; Sawhney, R. L. Effect of storage on thermal performance of a building. International Journal of Energy Research, 16, 1992, p 697-707.

11. Bzowska, D. Stochastic modelling of thermal performance for a heated building. Archives of Civil Engineering, 4, 1997.

12. Spitler, J. D.; Ferguson, J. D. Overview of the ASHRAE annotated guide to load calculations models and algorithms. ASHRAE Transactions, Vol 102, 1996, No 1; Vol 101, 1995, No 2.

13. Koczyk, H. Analysis of thermal states of buildings needed for energy economical heating. Politechnika Poznańska, Rozprawa, No 241, 1990, p 138 (in Polish).

14. Koczyk, H. Analysis of heating maintenance parameters using the matrix method, Civil Engineering (Statyba), No 2, Vilnius: Technika, 1996, p 68-72. 